\section{H-Y minor histocompatibility antigen influences renal allograft survival}

The male-specific minor histocompatibility antigen $\mathrm{H}-\mathrm{Y}$ is well known to affect the success of hematopoietic stem-cell transplantation; therefore, the sex of the donor and recipient is routinely taken into account in pretransplantation risk assessments. Since $\mathrm{H}-\mathrm{Y}$ is expressed in all tissues, this antigen would also be expected to have a role in graft rejection after renal transplantation. Registry analyses have shown, however, that the survival of kidneys from male donors is not reduced in female recipients.

To further investigate this issue, Gratwohl and colleagues retrospectively reviewed data from 158,652 adults in 45 countries who had undergone deceased-donor kidney-only transplantation during 1985-2004 (mean follow-up $5.6 \pm 4.8$ years). According to univariate analysis of the four possible donor-recipient sex combinations, transplantation of kidneys from female donors into male recipients was associated with the poorest graft survival. However, on multivariate analysis, the risk of graft failure was highest for male kidneys in female recipients; hazard ratios in this group were 1.08 (95\% Cl 1.03-1.14; $P=0.003$ ) for graft loss during the first year after transplantation and 1.06 (95\% Cl 1.01-1.10; $P=0.008)$ for graft loss during the subsequent 9 years.

These data indicate that donor and recipient sex should be taken into account during allocation of donor kidneys. The authors suggest that a compensatory effect of a higher nephron dose in male kidneys might explain why univariate analyses apparently fail to show that male donor kidneys fare worse in female recipients.

Original article Gratwohl A et al. (2008) H-Y as a minor histocompatibility antigen in kidney transplantation: a retrospective cohort study. Lancet 372: 49-53

\section{Sirolimus might cause diabetes and reduce male fertility after renal transplantation}

Results from two retrospective studies have now added to the mounting evidence that sirolimus is associated with serious adverse events.

Johnston et al. analyzed data from the United States Renal Data System on 20,124 nondiabetic adult recipients of a first, kidneyonly transplant. During follow-up (median 2.63 years), patients treated with sirolimus plus ciclosporin, sirolimus plus tacrolimus, or sirolimus plus mycophenolate mofetil or azathioprine were at increased risk of new-onset diabetes (hazard ratios 1.61, $P<0.0001,1.66$, $P<0.0001$ and 1.36, $P<0.01$, respectively) compared with patients who received ciclosporin plus mycophenolate mofetil or azathioprine.

Zuber et al. analyzed questionnaire responses, telephone conversations or face-to-face consultations with 95 men (aged 20-40 years) who underwent renal transplantation during the period 1995-2005. Spontaneous conception and pregnancies were recorded for the partners of 1 of the 17 patients who received continuous sirolimus, 22 of the 52 patients who did not receive sirolimus, and 12 of the 26 patients who received intermittent sirolimus; mean followup in the three groups was $5.6 \pm 2.5$ years, $5.0 \pm 3.4$ years and $6.9 \pm 3.3$ years, respectively. Partners of patients who received sirolimus had a lower spontaneous conception rate than those who were not given sirolimus (5.9 per 1,000 patient-years vs 92.9 per 1,000 patientyears; $P=0.007$ ). Analysis of sperm from 19 patients showed that continuous sirolimus use was associated with a significantly lower sperm count than nonuse of sirolimus $\left(28.6 \pm 31.2 \times 10^{6}\right.$ vs $\left.292.2 \pm 271.2 \times 10^{6} ; P=0.006\right)$.

Original articles Johnston O et al. (2008) Sirolimus is associated with new-onset diabetes in kidney transplant recipients. J Am Soc Nephrol 19: 1411-1418

Zuber J et al. (2008) Sirolimus may reduce fertility in male renal transplant recipients. Am J Transplant 8: 1471-1479 